\title{
Curcumin regulates the metabolism of low density lipoproteins by improving the C-to-U RNA editing efficiency of apolipoprotein $B$ in primary rat hepatocytes
}

\author{
NAN TIAN ${ }^{1}$, XIAOLING LI $^{1}$, YANG LUO ${ }^{1}$, ZIWU HAN $^{1}$, ZHAOHUI LI $^{2}$ and CHUNLEI FAN ${ }^{1}$ \\ ${ }^{1}$ Department of Cell Biology, College of Life Science, Zhejiang Chinese Medical University, Hangzhou, Zhejiang 310053; \\ ${ }^{2}$ Department of Neurosurgery, China-Japan Union Hospital, Jilin University, Changchun, Jilin 130031, P.R. China
}

Received June 8, 2013; Accepted October 7, 2013

DOI: $10.3892 / \mathrm{mmr} .2013 .1754$

\begin{abstract}
There are two isoforms of apolipoprotein B (apoB) in mammals: apoB-100 and apoB-48. The latter is generated by $\mathrm{C}$-to- $\mathrm{U}$ editing of apoB mRNA, catalyzed by the apolipoprotein B mRNA editing enzyme, namely, catalytic polypeptide 1 (APOBEC-1). Lipid particles containing apoB-48 are cleared from the plasma more rapidly than those containing apoB-100 and thus do not contribute to plaque formation in the arterial wall. In the present study, we analyzed whether curcumin is capable of regulating lipid metabolism by improving the level of apoB mRNA editing. The cytotoxicity of curcumin in hepatocytes was determined using the 3-(4,5-dimethylthiazol-2-yl)-2,5-diphenyltetrazolium bromide (MTT) assay and the levels of APOBEC-1 mRNA and protein were analyzed by real-time quantitative polymerase chain reaction (qRT-PCR) and western blotting. The efficiency of apoB mRNA editing was determined by reverse transcription PCR (RT-PCR) products and cloning sequencing analysis. We demonstrated that curcumin concentrations up to $70 \mu \mathrm{M}$ had no significant cytotoxic effects on primary rat hepatocytes at $24 \mathrm{~h}$. At $15 \mu \mathrm{M}$, curcumin significantly increased the expression of APOBEC-1 mRNA and protein, and increased the editing level of apoB mRNA from 3.13 to $7.53 \%$. At $25 \mu \mathrm{M}$, curcumin reduced the expression of APOBEC-1; however, it did not affect the apoB mRNA editing level. Our data suggested that curcumin at a concentration of $15 \mu \mathrm{M}$ raised the level of apoB-48 and reduced the level of apoB-100 by increasing the expression of APOBEC-1 in primary rat hepatocytes; therefore, curcumin may be a novel preventative therapy for atherosclerosis.
\end{abstract}

Correspondence to: Professor Chunlei Fan, Department of Cell Biology, College of Life Sciences, Zhejiang Chinese Medical University, 548 Binwen Road, Hangzhou, Zhejiang 310053, P.R. China

E-mail: 1x15544@126.com

Key words: curcumin, mRNA editing, ApoB-48, primary rat hepatocytes, atherosclerosis

\section{Introduction}

Apolipoprotein B (ApoB), a major component of low-density lipoproteins (LDLs), is important in lipid metabolism. It is responsible for the transport of LDLs and mediates their absorption by cells through LDL receptor-mediated endocytosis. High plasma LDL levels contribute to the formation of plaques in arterial walls, which may eventually block arteries and cause cardiovascular disease $(1,2)$.

In mammals, there are two isoforms of apoB: apoB-100 and apoB-48. The two isoforms are encoded by a single mRNA transcript. The cytidine residue $(\mathrm{C})$ at nucleotide 6666 of the apoB mRNA is edited to a uracil (U) by the apolipoprotein B mRNA editing enzyme APOBEC-1. This $\mathrm{C}$-to-U editing generates a UAA translational stop codon, resulting in the truncated gene product known as apoB-48 (3). ApoB-48 lacks apoB-100's C-terminal LDL receptor binding region and is unable to bind to LDL receptors. However, lipoprotein particles containing apoB-48 possess multiple copies of apolipoprotein E, which assists with the clearance of particles through LDL receptors and LDL receptor-related proteins $(4,5)$. Lipoprotein particles containing apoB-48 clear from plasma more rapidly than those containing apoB-100 (10 min compared with 3 days) (6). In theory, elevating the expression of APOBEC-1 in the liver should increase the efficiency of apoB mRNA editing, leading to a greater secretion of lipoproteins containing apoB-48, a lower concentration of apoB-100 LDLs in the plasma and reduced formation of atherosclerotic plaques.

Curcumin, an extract from the traditional Chinese medicinal herb Curcuma longa L., possesses numerous important biological activities. It has potential as a chemotherapeutic agent and has hypocholesterolemic properties, lowering the level of LDL-cholesterol in plasma (7-9). In the present study, we used primary rat hepatocytes as a model system to investigate whether the expression of APOBEC-1 and the level of apoB mRNA editing may be improved by curcumin treatment. Through this study, we aimed to identify a potential new therapy to increase the amount of apoB-48 and reduce the amount of apoB-100 in order to lower the incidence of atherosclerosis. Furthermore, changes in APOBEC-1 expression levels and apoB mRNA editing efficiency were analyzed 
to gain insight into the molecular mechanisms of lipid metabolism underlying the effects of curcumin.

\section{Materials and methods}

Animals. Animal experimental procedures were performed in conformity with the Guide for the Care and Use of Laboratory Animals (NIH publication no. 85-23, revised 1996) and were approved by the Ethics Committee of the Animal Laboratory Research Centre of Zhejiang Chinese Medical University [Zhejiang, China; approval no. SYXK (Zhe) 2008-0115]. Sprague-Dawley (SD) rats within $24 \mathrm{~h}$ of birth were purchased from Shanghai B\&K Universal Group Limited (Shanghai, China). Standard laboratory food and water were available ad libitum.

Isolation of primary rat hepatocytes. Primary hepatocytes were obtained from male SD rats within $24 \mathrm{~h}$ of birth. We used an improved collagenase/trypsin digestion method, as follows: Rats were decapitated and the livers were extracted and placed in cold D-Hanks solution. Liver capsules were peeled off with forceps and the livers were cut into small pieces with ophthalmic scissors. Tissues were thoroughly washed and then digested with $0.25 \mathrm{mg} / 1$ trypsin for $3 \mathrm{~min}$ at $37^{\circ} \mathrm{C}$. Dulbecco's modified Eagle's medium (DMEM) plus $10 \%$ fetal bovine serum (FBS) was added to terminate the digestion process. The digested tissues were centrifuged at $800 \mathrm{~g}$ at $15-25^{\circ} \mathrm{C}$ for $5 \mathrm{~min}$ and the supernatant was removed. Tissues were transferred to a sterile glass bottle and digested for 15 min using $0.2 \mathrm{mg} / 1$ type IV collagenase. The reaction was terminated by adding DMEM plus $10 \%$ FBS until the digested material became flocculent. The dispersed cells were filtered through a 200-mesh sieve and centrifuged for $5 \mathrm{~min}$ at $800 \mathrm{~g}$. The cell pellet was washed twice with fresh medium and the differential adhesion method was used to remove the fibroblasts. Cell viability was $>95 \%$, which was measured using the trypan blue exclusion test.

Culturing and identification of primary rat hepatocytes. Primary rat hepatocytes (viability $>95 \%$ ) were seeded in culture flasks at a density of $4 \times 10^{5}$ to $5 \times 10^{5}$ cells $/ \mathrm{ml}$ and incubated in DMEM/F12 plus 5\% FBS. The day prior to identification, the cells $\left(5 \times 10^{4}\right.$ cells $\left./ \mathrm{ml}\right)$ were plated onto glass cover slips in 24-well dishes and grown for $24 \mathrm{~h}$. Cells were washed twice with phosphate buffer saline (PBS; pH 7.4) and then stained using a periodic acid-Schiff kit according to the manufacturer's instructions (Nanjing Jiancheng Bioengineering Institute, Nanjing, Jiangsu, China). Stained cells were mounted on glass slides and images were captured using a Nikon Eclipse 80i microscope connected to a DS-5M-L1 camera.

Cell viability assay. Cell viability of primary rat hepatocytes was measured using the MTT viability test. Briefly, cells were seeded at a density of $6 \times 10^{3}$ cells/well in 96 -well plates with or without increasing concentrations of curcumin for $24 \mathrm{~h}$. MTT $(50 \mu \mathrm{l}, 2 \mathrm{mg} / \mathrm{ml})$ was added to each well and the plate was incubated for an additional $4 \mathrm{~h}$. Following removal of the medium, formazan crystals were dissolved in $150 \mu \mathrm{l}$ of dimethyl sulfoxide (DMSO). The absorbance of MTT-formazan at $550 \mathrm{~nm}$ was measured using a SpectraMaxM3 microplate reader.

Real-time PCR analysis. Following treatment of primary rat hepatocytes with no curcumin or with 5,15 or $25 \mu \mathrm{M}$ curcumin for $24 \mathrm{~h}$, total RNA was isolated using TRIzol reagent (Invitrogen Life Technologies, Carlsbad, CA, USA) according to the manufacturer's instructions. Complementary DNA (cDNA) was synthesized using a HiFi-MMLV cDNA kit (Beijing Kang Century Biotechnology Co., Ltd., Beijing, China). qRT-PCR was conducted using UltraSYBR mixture (Beijing Kang Century Biotechnology Co., Ltd.). All primers were designed using primer5 (Premier Biosoft, Palo Alto, CA, USA). Primers for the $\beta$-actin gene were 5'-GGCACCACACCTTCTACAAT-3' (forward) and 5'-GTGGTGGTGAAGCTGTAGCC-3' (reverse).Primersforthe APOBEC-1 gene were 5'-GAGCCCCACGAGTTTGAAGT-3' (forward) and 5'-ACACCGCTGCTAATAAGGTC-3' (reverse). All samples were run in triplicate and changes in gene expression were calculated using the $\Delta \Delta \mathrm{Ct}$ method. For quantitative analysis of apoB mRNA editing, a regular polymerase chain reaction (PCR) was performed using Taq DNA polymerase (Beijing Kang Century Biotechnology Co., Ltd.) and primers 5'-GGCTTCCTCAGCAGATTCAT-3' (forward) and 5'-ATCCAAGACGCACCACTACT-3' (reverse).

Western blot analysis. Proteins from primary rat hepatocytes from the control group (cultured for $24 \mathrm{~h}$ without curcumin) and the treatment groups (cultured for $24 \mathrm{~h}$ with 5,15 or $25 \mu \mathrm{M}$ curcumin) were extracted using Protein Extraction Reagent (Boster Bioengineering, Wuhan, China) containing $1 \mathrm{mM}$ phenylmethanesulfonyl fluoride (PMSF; Roche Molecular Biochemicals, Indianapolis, IN, USA). Protein concentrations were determined using the bicinchoninic acid (BCA) protein assay (Nanjing KeyGen Biotech Co. Ltd., Nanjing, Jiangsu, China). The proteins were separated by $10 \%$ SDS-PAGE and then transferred onto a polyvinylidene difluoride (PVDF) membrane (Pall Gelman Laboratory Corporation, Ann Arbor, MI, USA). For western blot analyses, membranes were blocked for $2 \mathrm{~h}$ in PBS containing 5\% nonfat dried milk powder at room temperature, incubated overnight with the primary antibody at $4^{\circ} \mathrm{C}$, washed, and then incubated with the secondary antibody for $2 \mathrm{~h}$ at room temperature. Chemiluminescence was detected using an EZ-ECL Chemiluminescence Detection kit for horseradish peroxidase (HRP; Biological Industries, Beit HaEmek, Israel). Mouse antibody to APOBEC-1 (Santa Cruz Biotechnology, Inc., Santa Cruz, CA, USA) and $\beta$-actin (Boster Bioengineering) and goat anti-mouse immunoglobulin G (IgG; Hangzhou HuaAn Biotechnology Co., Ltd., Hangzhou, Zhejiang, China) were used in this study.

Quantification of RNA editing level. The editing level of apoB mRNA $\mathrm{C}^{6666}$ sites was determined from mixed $\mathrm{C} / \mathrm{T}$ peaks at site 6666 in RT-PCR production sequence chromatograms, where the genomically encoded base was cytidine. The ratio between the $\mathrm{C}$ and $\mathrm{T}(\mathrm{U})$ peak heights in individual chromatograms was measured using FinchTV. Percent editing was calculated as the $\mathrm{T}$ peak height relative to the total peak height $(\mathrm{T} /(\mathrm{C}+\mathrm{T}) \times 100)$. Since this method is not quantitative, we also assessed editing levels by counting clones. We cloned 
the RT-PCR products containing the edited cytidine into the pUC-T vector (Beijing Kang Century Biotechnology Co., Ltd.). For each sample, 90 clones were sequenced by Shanghai Sangon Biological Engineering Technology \& Services Co., Ltd. (Shanghai, China).

Statistical analysis. Statistical analysis was performed using one-way analysis of variance (ANOVA) for multiple comparisons and $\mathrm{t}$-tests among groups. $\mathrm{P}<0.05$ was considered to indicate a statistically significant difference and $\mathrm{P}<0.01$ was considered to indicate an extremely statistically significant difference.

\section{Results}

Curcumin has no significant effect on the viability of primary rat hepatocytes. The cells isolated from newborn SD rats had a rounded orpolygonal cell body with a translucent cytoplasm and nearly covered the bottom of the flask following 5 days of culture in DMEM/F12 (Fig. 1A). Following periodic acidSchiff staining, dense pink glycogen granules were observed in the cytoplasm of the cells. Vacuoles were present around nuclei, which were stained blue with hematoxylin (Fig. 1B). These observations indicated that plenty of pure rat hepatocytes were harvested using the improved collagenase/trypsin digestion method. These pure rat hepatocytes were used in all the following experiments within three generations. The viability of hepatocytes treated with 10 to $70 \mu \mathrm{M}$ curcumin was not significantly different from that of the control group (Fig. 2A), indicating that curcumin concentrations up to $70 \mu \mathrm{M}$ have no significant cytotoxic effects on these cells. Therefore, test concentrations of 5,15 and $25 \mu \mathrm{M}$ were selected. We also studied the effect of $25 \mu \mathrm{M}$ curcumin at different time points. MTT assays revealed that while treatment for $0-24 \mathrm{~h}$ had no effect on hepatocyte viability, cell viability was reduced following $36 \mathrm{~h}$ of exposure (Fig. 2B). Therefore, primary rat hepatocytes were treated with 5,15 or $25 \mu \mathrm{M}$ curcumin for $24 \mathrm{~h}$.

Curcumin promotes the expression of APOBEC-1 in a non dose-dependent manner. To obtain insights into the effect of curcumin on apoB mRNA editing efficiency, we first evaluated the change in APOBEC-1 mRNA expression following curcumin treatment by qRT-PCR analysis of RNA samples from primary rat hepatocytes from the control group and the treatment groups (Fig. 3A). APOBEC-1 mRNA levels were up to more than three-fold higher in cells treated with $15 \mu \mathrm{M}$ curcumin than in untreated cells; however, mRNA levels were markedly reduced in cells treated with $25 \mu \mathrm{M}$ curcumin. Similar results were obtained when APOBEC-1 protein levels were analyzed by western blotting (Fig. 3B). These results indicate that curcumin affects the expression of APOBEC-1 in primary rat hepatocytes; however, not in a dose-dependent manner.

Curcumin $(15 \mu M)$ increases the efficiency of apoB $m R N A$ editing. To evaluate the functional role of curcumin, apoB mRNA editing levels in all groups were first investigated by sequencing apoB mRNA RT-PCR products. As shown in Fig. 4A, the C-to-U editing signal was only able to be detected

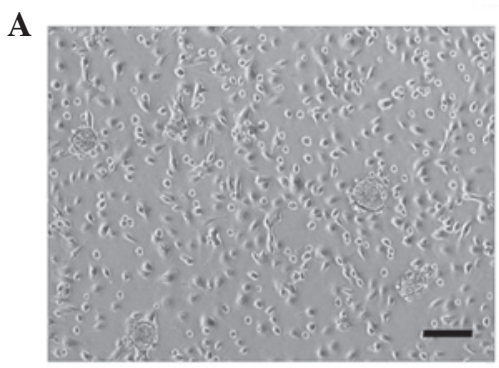

B

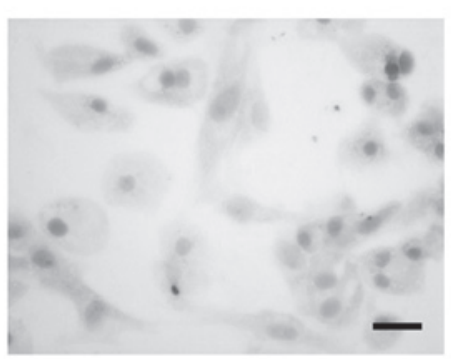

Figure 1. Identification of primary rat hepatocytes by PAS. (A) Cultured primary rat hepatocytes after 5 days (post-isolation). (B) PAS reaction showed the dense glycogen granules in primary rat hepatocytes' cytoplasm. Magnifications: (A) bar $=20 \mu \mathrm{m}$; (B) bar $=10 \mu \mathrm{m}$. PAS, periodic acid-Schiff stain

A

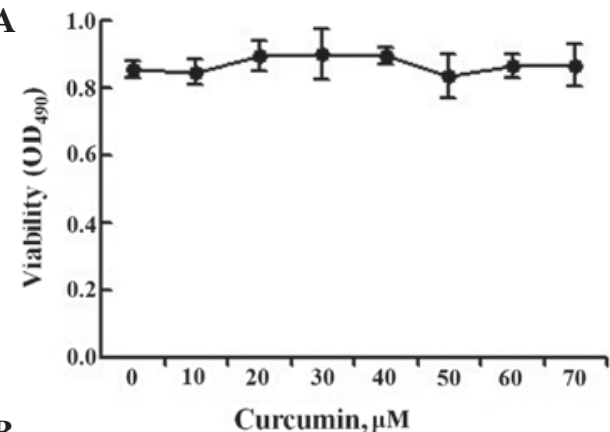

B

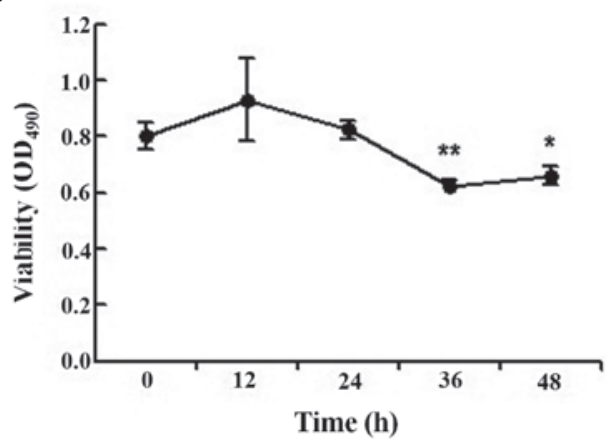

Figure 2. Primary rat hepatocytes' viability was determined by the MTT assay. (A) Cells were exposed to curcumin at different concentrations. (B) Cells were exposed to $25 \mu \mathrm{mol} / 1$ curcumin for different times $\left({ }^{*} \mathrm{P}<0.05\right.$, $\left.{ }^{* *} \mathrm{P}<0.01\right)$. OD490, optical density values at $490 \mathrm{~nm}$; MTT, 3-(4,5-dimethylthiazol-2-yl)-2,5-diphenyltetrazolium bromide.

in the $15 \mu \mathrm{M}$ curcumin treatment group. We also demonstrated that the apoB mRNA editing efficiency was significantly higher in newborn rat liver tissues than in the primary rat hepatocytes. To assess editing levels more quantitatively, we sequenced at least $3 \times 30$ clones from each group (Fig. 4B). The apoB mRNA editing level increased from $3.13 \%$ in the control group (Fig. 4A, hepatocytes control) to $7.53 \%$ in the $15 \mu \mathrm{M}$ curcumin 


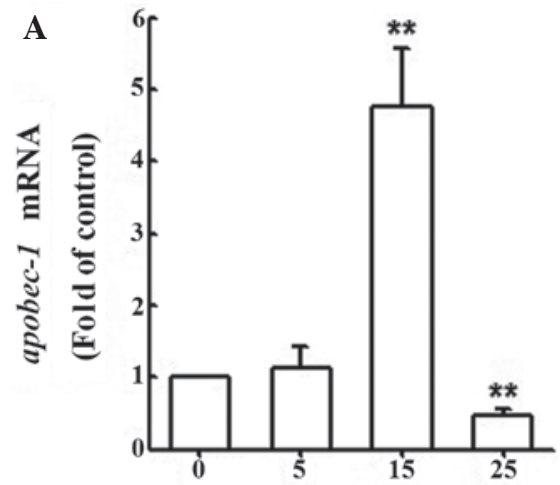

B

Curcumin, $\mu \mathrm{M}$

Figure 3. Effect of curcumin on APOBEC-1 expression with different concentrations. (A) Effect of curcumin on APOBEC-1 RNA levels in primary rat hepatocytes ( $(" \mathrm{P}<0.05)$. (B) Effect of curcumin on APOBEC-1 protein levels in primary rat hepatocytes. Rat primary hepatocytes were prepared and treated with no curcumin or with 5,15 and $25 \mu \mathrm{M}$ curcumin for $24 \mathrm{~h}$, followed by western blotting with indicated antibody. All panels show the representative blot $(\mathrm{n}=3)$. APOBEC-1, apolipoprotein B mRNA editing enzyme catalytic polypeptide-1.

A

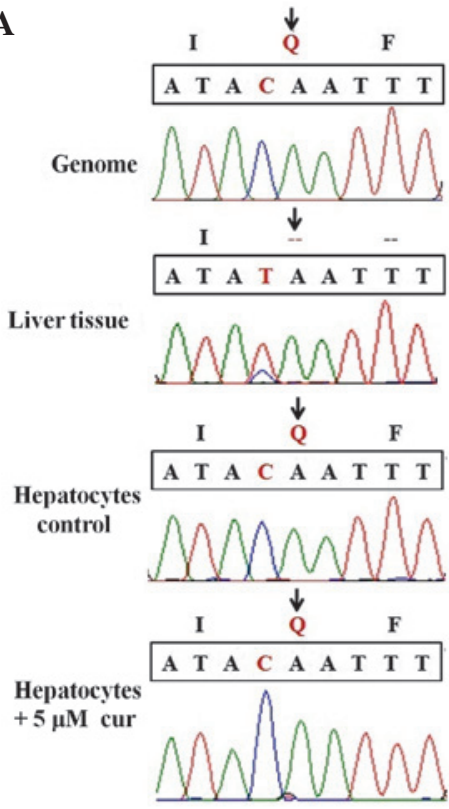

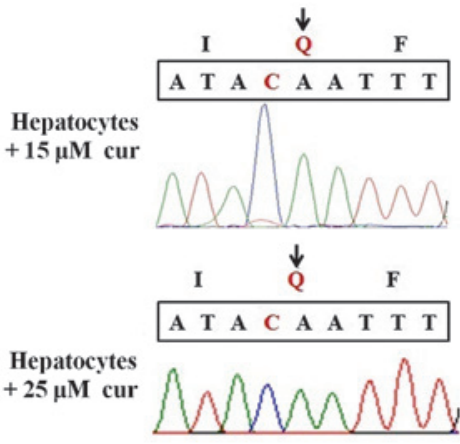

B

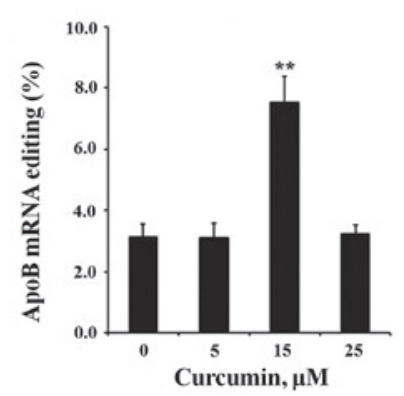

Figure 4. Comparison of apoB mRNA editing efficiency in cur treatment groups and the control group. (A) Detection of the apoB $\mathrm{C}^{6666}$ editing site in primary rat hepatocytes by RT-PCR production sequencing. (B) Quantitative analyses of apoB editing efficiency. Data demonstrated that the editing levels in the $15 \mu \mathrm{M}$ cur group increased significantly $(\mathrm{P}<0.01)$. ApoB, apolipoprotein $\mathrm{B}$; cur, curcumin; RT-PCR, reverse transcription-PCR.

treatment group; however the other curcumin concentrations had no significant effect. Furthermore, we discovered no other editing site in the apoB mRNA besides the normal $\mathrm{C}^{6666}$ site, in all groups. From these analyses, we concluded that $15 \mu \mathrm{M}$ curcumin increased the amount of apoB-48 and reduced the amount of apoB-100 in primary rat hepatocytes.

\section{Discussion}

Previous studies have demonstrated that curcumin is able to reduce the LDL concentration in plasma in several different ways, for example by upregulating the LDL receptor via the SCAP-SREBP pathway to eliminate LDL-cholesterol in the blood (10); by causing significant activation of liver $\mathrm{X}$ receptor
(LXR) and scavenger receptor class A (SR-A) to decrease LDL-cholesterol synthesis (11) and by elevating the activity of hepatic cholesterol- $7 \alpha$-hydroxylase, which is a key enzyme in cholesterol degradation (12). However, in the present study, we focused on the editing of the mRNA transcript encoding apoB, the primary apolipoprotein of 'bad cholesterol', and verified the LDL-cholesterol-lowering effect of curcumin in a new way.

We used primary rat hepatocytes, isolated from newborn SD rats by an improved collagenase/trypsin digestion method, as a model system. Since primary cultured cells maintain the basic properties of the original cells, they are extremely good biological models for drug experiments (13). To investigate the effect of curcumin, we performed qRT-PCR and western blot analyses to detect the expression levels of APOBEC-1 in 
primary hepatocytes treated with different concentrations of curcumin. In addition, an apoB mRNA fragment covering $\mathrm{C}^{6666}$ was PCR amplified from cDNA and cloned, and then the PCR products and the clones were sequenced to determine differences in the mRNA editing efficiency between curcumintreated and untreated cells. The results clearly showed that $15 \mu \mathrm{M}$ curcumin increased the expression of APOBEC-1 at the mRNA and protein levels; however, $25 \mu \mathrm{M}$ curcumin reduced the expression of this cytidine deaminase. Therefore, curcumin increased the expression of APOBEC-1 in a non dose-dependent manner. Furthermore, when the expression of APOBEC-1 increased, the apoB mRNA editing level also increased (significant result, $\mathrm{P}<0.01$ ). In conclusion, our study demonstrated that an intermediate concentration of curcumin increased the level of apoB mRNA editing by increasing the expression of APOBEC-1, which increased apoB-48 and reduced apoB-100.

Other studies have demonstrated that adenoviral expression of exogenous APOBEC-1 is able to effectively increase apoB mRNA editing efficiency and reduce plasma LDL levels; however, the brief duration of transgene expression and the immune response to adenoviral vectors make this method unsuitable for studies on atherosclerosis (14). Curcumin is a natural compound and has been demonstrated to be non toxic to humans at dosages of up to $8000 \mathrm{mg} /$ day when administered orally for 3 months (15). Therefore, compared with the adenoviral delivery method, curcumin has the potential to increase apoB mRNA editing efficiency for a longer period without toxic side effects.

Transgenic animals provide another approach that is capable of yielding long-term increases in APOBEC-1 expression in the liver; however, hypermutation that is believed to induce liver dysplasia and hepatocellular carcinomas through hyperediting of apoB and other mRNAs has been discovered in such transgenic animals (16). Therefore, there remains numerous obstacles to the use of gene therapy for the clinical treatment of atherosclerosis. In the present study, no editing site other than the normal $\mathrm{C}^{6666}$ site was detected in primary rat hepatocytes treated with $15 \mu \mathrm{M}$ curcumin. Patterson et al found that APOBEC-1 increased apoB mRNA hyperediting in a dose-dependent manner, such that when the apoB mRNA editing level was higher than $10 \%$, additional editing sites were discovered. In the present study, the $\mathrm{C}^{6666}$ site editing level in the $15 \mu \mathrm{M}$ treatment group increased to only $7.53 \%$. As the editing level did not exceed $10 \%$, hyperediting may have been avoided even though curcumin increased the expression level of APOBEC-1. In conclusion, compared with adenoviral delivery and transgenic animals, curcumin treatment has clear advantages for therapeutic use to reduce atherogenic lipoprotein levels and prevent atherosclerosis.

C-to-U editing of apoB mRNA is mediated not only by APOBEC-1, but also by other proteins that form an enzyme complex with APOBEC-1. Curcumin may also affect the expression of other components in this system, possibly explaining why $25 \mu \mathrm{M}$ curcumin did not affect the apoB mRNA editing level despite reducing the expression of APOBEC-1. Future studies of this mRNA editing system are required in order to further understand how curcumin increases the level of apoB mRNA editing.
To the best of our knowledge, this study is the first to state that curcumin increases the level of apoB-48 and reduces the level of apoB-100 through the C-to-U RNA editing enzyme APOBEC-1 in primary rat hepatocytes without hyperediting and toxicity. Compared with adenoviral delivery and transgenic animals, curcumin treatment has clear advantages, therefore it may be used therapeutically to reduce levels of atherogenic lipoproteins and prevent atherosclerosis.

\section{Acknowledgements}

This study was supported by research grants from the Natural Science Foundation of Zhejiang Province, Youth Fund Project (no. LQ12C07001).

\section{References}

1. Young SG: Recent progress in understanding apolipoprotein B. Circulation 82: 1574-1594, 1990.

2. Innerarity TL, Borén J, Yamanaka $\mathrm{S}$ and Olofsson SO: Biosynthesis of apolipoprotein B48-containing lipoproteins. Regulation by novel post-transcriptional mechanisms. J Biol Chem 271: 2353-2356, 1996.

3. Anant S, Blanc V and Davidson NO: Molecular regulation, evolutionary, and functional adaptations associated with $\mathrm{C}$ to $\mathrm{U}$ editing of mammalian apolipoprotein B mRNA. Prog Nucleic Acid Res Mol Biol 75: 1-41, 2003.

4. Anant S and Davidson NO: Molecular mechanisms of apolipoprotein B mRNA editing. Curr Opin Lipidol 12: 159-165, 2001.

5. Pitas RE, Innerarity TL and Mahley RW: Cell surface receptor binding of phospholipid protein complexes containing different ratios of receptor active and inactive E apoprotein. J Biol Chem 255: 5454-5460, 1980.

6. Innerarity TL and Mahley RW: Enhanced binding by cultured human fibroblasts of apo-E-containing lipoproteins as compared with low density lipoproteins. Biochemistry 17: 1440-1447, 1978.

7. Gupta SC, Patchva S, Koh W and Aggarwal BB: Discovery of curcumin, a component of the golden spice, and its miraculous biological activities. Clin Exp Pharmacol Physiol 39: 283-299, 2012.

8. Joe B, Vijaykumar M and Lokesh BR: Biological properties of curcumin-cellular and molecular mechanisms of action. Crit Rev Food Sci Nutr 44: 97-111, 2004.

9. Egan ME, Pearson M, Weiner SA, et al: Curcumin, a major constituent of turmeric, corrects cystic fibrosis defects. Science 304: 600-602, 2004.

10. Dou X, Fan C, Wo L, Yan J, Qian Y and Wo X: Curcumin up-regulates LDL receptor expression via the sterol regulatory element pathway in HepG2 cells. Planta Med 74: 1374-1379, 2008.

11. Zhao JF, Ching LC, Huang YC, et al: Molecular mechanism of curcumin on the suppression of cholesterol accumulation in macrophage foam cells and atherosclerosis. Mol Nutr Food Res 56: 691-701, 2012.

12. Srinivasan K and Sambaiah K: The effect of spices on cholesterol 7 alpha-hydroxylase activity and on serum and hepatic cholesterol levels in the rat. Int J Vitam Nutr Res 61: 364-369, 1991.

13. Wang $K$, Shindoh $H$, Inoue $T$ and Horii I: Advantages of in vitro cytotoxicity testing by using primary rat hepatocytes in comparison with established cell lines. J Toxicol Sci 27: 229-237, 2002.

14. Teng B, Blumenthal S, Forte T, et al: Adenovirus-mediated gene transfer of rat apolipoprotein $\mathrm{B}$ mRNA-editing protein in mice virtually eliminates apolipoprotein B-100 and normal low density lipoprotein production. J Biol Chem 269: 29395-29404, 1994.

15. Cheng AL, Hsu CH, Lin JK, et al: Phase I clinical trial of curcumin, a chemopreventive agent, in patients with high-risk or pre-malignant lesions. Anticancer Res 21: 2895-2900, 2001.

16. Hersberger M, Patarroyo-White S, Qian X, et al: Regulatable liver expression of the rabbit apolipoprotein B mRNA-editing enzyme catalytic polypeptide 1 (APOBEC-1) in mice lacking endogenous APOBEC-1 leads to aberrant hyperediting. Biochem J 369: 255-262, 2003. 